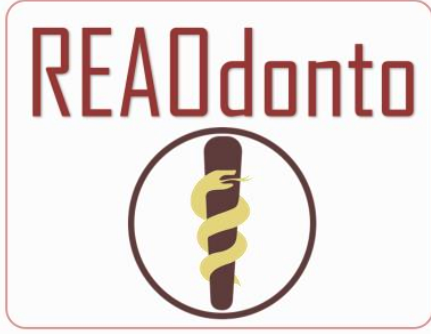

Revista Eletrônica Acervo Odontológico
REVISÃO BIBLIOGRÁFICA

Recebido em: 11/2019

Aceito em: $2 / 2020$

Publicado em: 3/2020

\title{
Aspectos psicossociais relacionados ao paciente desdentado: Uma revisão da literatura
}

\author{
Psychosocial aspects related to the deentified patient: A literature review
}
Aspectos psicosociales relacionados con el paciente deentificado: Una revisión de la literatura

\begin{abstract}
Mayara Monique Silva de Oliveira ${ }^{1}$, Tatyana Maria Carvalho Pereira Farias ${ }^{1}$, Rafaella de Souza Leao ${ }^{1}$, Rayanna Thayse Florêncio Costa ${ }^{1}$, Pedro Alves Muniz¹, Sandra Lúcia Dantas de Moraes.
\end{abstract}

Resumo: $O$ presente artigo teve como objetivo realizar uma análise das possíveis consequências psicológicas e sociais provocadas em consequência da perda dos elementos dentários. Para isso, uma busca eletrônica por artigos datados entre os anos de 2009 a 2019 foi realizada nas bases de dados: PUBMED, Scielo e o Google scholar. Dentre eles, 15 artigos foram incluídos para análise quantitativa e qualitativa, de acordo com os critérios de inclusão adotados. A perda dos dentes provocada por doenças bucais, falta de acesso à assistência em saúde, ausência de informação, condição socioeconômica, fatores culturais, bem como o modelo predominantemente mutilador, influência nos aspectos psicossociais que podem, por consequência, impactar na qualidade de vida dos indivíduos, pois tais condições interferem na alimentação, fonação e estética do paciente causando-Ihe dor e desconforto psicológico, e comprometendo a sua interação no meio social. Além disso, o edentulismo interfere na autoimagem e relações interpessoais, assim como no psicológico do indivíduo, causando sentimentos de constrangimento social, diminuição da autoconfiança, tristeza e baixa autoestima.

Palavras-chave: Arcada edêntula, Qualidade de vida, Prótese.

Abstract: This article aimed to carry out an analysis of the possible psychological and social consequences caused by the loss of dental elements. For this, an electronic search for articles dated between 2009 and 2019 was carried out in the databases: PUBMED, Scielo and Google scholar. Among them, 15 articles were included for quantitative and qualitative analysis, according to the inclusion criteria adopted. The loss of teeth caused by oral diseases, lack of access to health care, lack of information, socioeconomic status, cultural factors, as well as the predominantly mutilating model, influence on psychosocial aspects that can, consequently, impact the quality of life of individuals, as these conditions interfere with the patient's diet, phonation and aesthetics causing him pain and psychological discomfort, and compromising his interaction in the social environment. In addition, edentulism interferes with self-image and interpersonal relationships, as well as the individual's psychological, causing feelings of social embarrassment, decreased self-confidence, sadness and low selfesteem.

Key words: Edentulous arcade, Quality of life, Prosthesis.

\footnotetext{
${ }^{1}$ Faculdade de Odontologia de Pernambuco - Universidade de Pernambuco (FOP/UPE). Camaragibe - PE.

*E-mail: fop.posgraduacao@upe.br
} 
Resumen: Este artículo tuvo como objetivo realizar un análisis de las posibles consecuencias psicológicas y sociales causadas por la pérdida de elementos dentales. Para ello, se realizó una búsqueda electrónica de artículos con fecha entre 2009 y 2019 en las bases de datos: PUBMED, Scielo y Google Scholar. Entre ellos, se incluyeron 15 artículos para análisis cuantitativos y cualitativos, de acuerdo con los criterios de inclusión adoptados. La pérdida de dientes causada por enfermedades orales, la falta de acceso a la atención médica, la falta de información, el estado socioeconómico, los factores culturales, así como el modelo predominantemente mutilante, influyen en los aspectos psicosociales que pueden, en consecuencia, afectar la calidad de vida de los pacientes. individuos, ya que estas condiciones interfieren con la dieta, la fonación y la estética del paciente, lo que le causa dolor e incomodidad psicológica y compromete su interacción en el entorno social. Además, el edentulismo interfiere con la autoimagen y las relaciones interpersonales, así como con la psicología del individuo, causando sentimientos de verguenza social, disminución de la confianza en sí mismo, tristeza y baja autoestima.

Palabras clave: Arcade edéntulo, Calidad de vida, Protesis.

\section{INTRODUÇÃO}

A vaidade e a preocupação com a aparência vêm aumentando gradativamente entre os indivíduos que buscam incessantemente alcançar padrões de beleza preestabelecidos pela sociedade. Os que são considerados belos recebem tratamento prefe rencial, encontrando desta forma maior facilidade em relacionamentos e maiores oportunidades de trabalho. Mesmo não sendo essa a principal forma de avaliação, nem a mais importante, ela pode influenciar na saúde do indivíduo (SOAIGHER KA, et al., 2016).

A Organização Mundial de Saúde (OMS), em 1948 conceituou a saúde como "um estado de completo bem-estar físico, mental e social e não apenas a ausência de doença ou enfermidade". A Constituição Federal (1988) estabeleceu, entre outras propostas, que a saúde é um direito social de cidadania e dever do estado. Esse, por sua vez, deve assegurar a integralidade nas ações de saúde bucal, sendo o responsável pela regulamentação, fiscalização e controle das ações e serviços de saúde (BRASIL, 2012).

Embora a saúde bucal seja declaradamente reconhecida como importante, uma considerável fração da população ainda não possui acesso aos serviços essências (SILVA MES, et al., 2010). O Sistema Único de Saúde (SUS) ainda apresenta dificuldades para o atendimento da população que necessita de assistência odontológica, sendo essa uma das principais causas que levam a perda precoce da estrutura dentária. A ausência do elemento dentário, além de provocar alterações morfológicas e funcionais, causam dor e desconforto psicológico, gerando impactos negativos na qualidade de vida que afetam a autoestima dos pacientes.

Decorrente dessa problemática, o edentulismo, definido pelo Ministério da Saúde (2006) como a ausência de dentes na cavidade oral é uma questão de saúde pública e representa uma das desigualdades sociais que caracterizam o Brasil (FONSECA LLV, et al., 2015). O Cirurgião-Dentista desempenha papel fundamental no tratamento dos pacientes, devendo voltar sua atenção não apenas para a saúde bucal, levando em consideração que a odontologia é uma combinação entre a formação científica, visão humanística da promoção da saúde e a habilidade técnica. Portanto é de sua responsabilidade manter o equilíbrio emocional do paciente diante do procedimento odontológico, constituindo um modelo humanizado de atendimento (GUERRA CT, et al., 2014).

O objetivo deste trabalho é identificar por meio de uma revisão de literatura os impactos psicológicos e sociais causados pela perda dos dentes e elencar suas causas e efeitos. Diante disso, a hipótese nula dessa revisão de literatura é que a perda dos elementos dentários não interfere na qualidade de vida dos pacientes.

\section{MÉTODOS}

Esta revisão foi construída mediante a análise de artigos indexados em bases de dados nacionais e internacionais. Os seguintes termos foram utilizados como estratégia de busca: autoimagem, impactos psicológicos, efeitos psicológicos, condições sociais, qualidade de vida, indicadores sociais, perda dentária, 
edentulismo, saúde bucal; ou em inglês, emotional effects, social conditions, quality of life, oral health, oral health impact profile, tooth loss, loss of confidence, edentulism. Adicionalmente foi realizado uma busca manual nos periódicos: "Revista do Departamento de Educação Física e Saúde" e do "Mestrado em Promoção da Saúde da Universidade de Santa Cruz do Sul / Unisc", "Revista Saúde Integrada", "Periodontology 2000", "Revista Brasileira de Ciências em Saúde", "Gerontology", e "Revista Equilíbrio Corporal e Saúde".

Foram estabelecidos critérios de inclusão, como: revisões sistemáticas, ensaios clínicos, revisões de literatura e estudos qualitativos; e exclusão: estudos em outra língua além da portuguesa e inglesa, estudos com ano inferior a 2009 e estudos que não avaliavam os impactos psicológicos e sociais. Para tanto, foram incluídos 15 artigos para análise quantitativa e qualitativa., entre 2009 e 2019, selecionados através do banco de dados PUBMED, Scielo (Scientific Electronic Library OnLine) e Google Scholar (Figura 1).

Figura 1 - Fluxograma dos estudos selecionados de acordo com os critérios de inclusão e exclusão
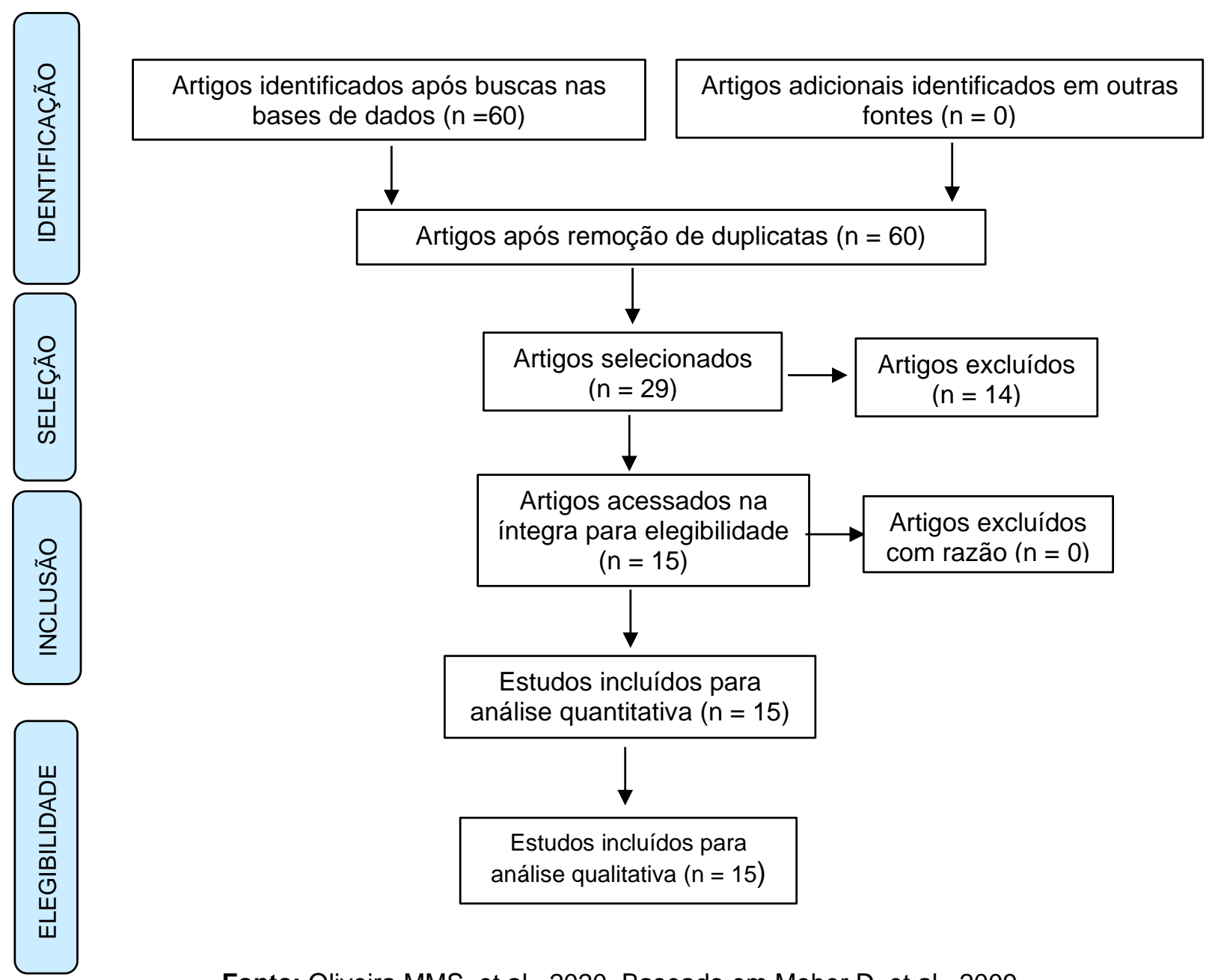

Fonte: Oliveira MMS, et al., 2020. Baseado em Moher D, et al., 2009.

\section{RESULTADOS E DISCUSSÃO}

\section{A cultura do Edentulismo no Brasil}

O edentulismo, definido como a perda total dos elementos dentários, representa um fenômeno marcante na vida do indivíduo e é visto como um aspecto determinante da condição de saúde bucal e das desigualdades sociais de uma população (FONSECA LLV, et al., 2015). Um levantamento epidemiológico realizado no Brasil, em 1986, apresentou os primeiros dados a respeito das condições de saúde bucal da população e foram 
obtidos números preocupantes em relação a quantidade de desdentados no brasil, revelando uma prática odontológica com enfoque no tratamento curativo, caracterizada por exodontias em massa.

Este cenário se devia à influência predominante do modelo curativo-mutilador oferecido pelos serviços odontológicos (CARNEIRO VFA, et al., 2012) que perdura até os dias de hoje. Além disso, a visão da prótese dentária (artefato utilizado para substituir as estruturas dentárias perdidas) vista como um símbolo de status socioeconômico e que garantia dentes alinhados e esteticamente favoráveis, influenciou para a sustentação desse modelo. (BORTOLI FR, et al., 2017)

A saúde bucal da população tem reflexo direto na saúde integral e na qualidade de vida de toda a sociedade (IBGE, 2013). A mutilação dentária, resultante da perda dos dentes é um fator predisponente a diversas doenças, pois provoca mudanças físicas, biológicas e emocionais nos indivíduos submetidos a essa prática (DOMINGOS MA, 2012). Apesar disso, diferente da perda de outras partes do corpo, o desdentado não é considerado doente ou deficiente. Culturalmente a população enxerga na remoção do elemento dentário uma solução para os problemas da saúde bucal (PROBST LF, et al., 2016).

A reduzida procura por serviços odontológicos, a ausência de medidas preventivas, as barreiras de acesso ao Sistema Único de Saúde (SUS), o diagnóstico e o tratamento inadequado e a fobia ao tratamento odontológico são motivos pelos quais as extrações dentárias eram priorizadas, mesmo existindo a possibilidade de recuperação da estrutura comprometida, através de métodos mais conservadores (DE SILVA MES, et al., 2010).

As causas que levam a degradação e consequente perda dos elementos, são os fatores biológicos e não biológicos. Dentre os fatores não biológicos pode-se apontar a regularidade da visita ao dentista, o estilo de vida, condições demográficas e socioeconômicas (BATISTA MJ, et al., 2012). Segundo Barbaro e Peres, 2015, o contexto socioeconômico influencia diretamente na perda dos elementos. Esses autores, observaram em seu estudo que quanto menos favoráveis são as condições sociais de um indivíduo, mais vulnerável ele se torna à perda dentária. Além disso, segundo Batista MJ, et al., (2012), dentre os fatores biológicos, a cárie e a doença periodontal, são os principais responsáveis pela destruição da estrutura dentária. Um estudo realizado por Carneiro VFA, et al. (2012) apontou a doença cárie como a principal responsável pela perda dos elementos dentários, sendo responsável por $47 \%$ das extrações entre os indivíduos avaliados. Domingos MÁ (2012) corroboram com essa informação, ao destacarem em seu estudo, que além da cárie, a doença periodontal também constitui a maior causa da perda dos dentes.

A junção dos fatores biológicos e não biológicos geram impacto estético, anatômico, biomecânico e social (OKOJE VN, et al., 2012) que afetam diretamente a qualidade de vida dos indivíduos, principalmente quando atinge seu bem-estar e condição nutricional (SAINTRAIN MV e DE SOUZA EH, 2012).

\section{Impactos psicológicos e sociais proveniente das perdas dentárias}

A perda dos dentes exerce impacto em três importantes âmbitos: social; emocional ou psicológico; funcional (SILVA MES, et al., 2010). Entretanto a preocupação com a reposição dos elementos perdidos é maior quando a estética está envolvida, e menor quando o restabelecimento da função dentária é necessário. (NEPOMUCENDO NVA, et al., 2018). A perda dos elementos dentários é uma situação marcante na vida de um indivíduo, pois causa mudanças em sua aparência facial, e como consequência, a fuga das situações sociais e medo de relações pessoais mais íntimas. A autoimagem, concebida através de referências de um padrão ideal imposto pela sociedade e preestabelecida desde o início da infância, está intimamente ligada à autoestima. Assim, uma modificação desse padrão, como por exemplo, a ausência dos dentes, leva a diminuição da autoestima do indivíduo (WOLF RMS, 2008; SCHULTHEISZ TSV e APRIL MRA, 2015).

A baixa autoestima dificulta o relacionamento das pessoas com a sociedade, principalmente com aqueles ao seu redor. De forma que ao vivenciarem relações afetivas mais íntimas, essas tendem a queixar-se e a cobrar afeto, consideração e respeito, como se as pessoas a sua volta não as valorizassem, tendendo a afasta-las. Essa reação defensiva é reflexo da insegurança formada pelos sentimentos de inferioridade e 
exclusão social, gerando uma instabilidade emocional que pode vim a interferir na saúde física e mental desses indivíduos (SCHULTHEISZ TSV e APRIL MRA, 2015)

Por outro lado, De Silva MES, et al. (2010) verificaram em seu estudo realizado com 50 indivíduos, que a perda dos dentes não interferiu significativamente na interação com as pessoas do seu convívio, nem as incapacitou de realizarem suas atividades cotidianas, todavia ocasionou um certo nível de inabilidade e desconforto psicológico, dor e algumas alterações na qualidade de vida. No entanto, Soaigher KA, et al. (2016) ressalta que os impactos gerados pela perda dos elementos dentários na qualidade de vida dependem da importância atribuída por cada indivíduo.

Alguns sinais clínicos característicos que podem ser observados em pacientes edêntulos, são: alterações no lábio, tendência a inversão, redução da largura da comissura labial acompanhada pelo aumento do ângulo nasolabial, cavitação das bochechas, sulcos e aparecimento de rugas. A extração dos dentes acarreta na redução de osso alveolar, que apresenta uma reabsorção contínua, porém não constante, na qual a crista alveolar perde um terço da sua altura nos primeiros 40 dias, sendo maior nos primeiros trimestres. Tal reabsorção implica em uma modificação no perfil e nas dimensões das arcadas levando a uma alteração nos músculos da expressão facial que sofrem distorção. Nos casos em que essa reabsorção se encontra mais avançada, estas são acompanhadas por uma redução em altura da face inferior e por um aumento da proeminência do queixo (ASMAR IE, 2017).

De acordo com Jorge TM, et al., (2009), os indivíduos que tiveram seus elementos dentários perdidos tendem a relatar frequente dificuldade na mastigação, devido a dependência direta para o corte e trituração dos alimentos. Uma das consequências dessas perdas é o prejuízo à saúde geral, tendo em vista que a dificuldade na mastigação pode interferir na escolha dos alimentos em consequência de sua consistência, 0 que, por sua vez, pode vim a comprometer o estado nutricional do indivíduo, bem como sua saúde. Além disso, a falta das estruturas dentárias também pode implicar em alterações do padrão articulatório, pois na ausência de dentes, a língua tende a se interpor na região desdentada com a finalidade de estabilizar a mandíbula, interferindo na fala, principalmente quando há ausência dos elementos anteriores, onde se nota omissão e substituição dos sons (JORGE TM, et al., 2009).

Domingos MÁ, et al. (2012) corrobora com Jorge TM, et al. (2009) ao afirmar que a perda dentária pode gerar consequências negativas para o estado nutricional do indivíduo, pois as alterações funcionais e mastigatórias podem interferir na formação do bolo alimentar. Entretanto no estudo de Jorge TM, et al. (2009), a perda dos elementos dentários não determinou a substituição dos alimentos, nem gerou problemas digestivos, contudo os indivíduos de sua pesquisa que apresentava ausência dentária relataram a presença de sensação dolorosa durante a mastigação.

Todos esses aspectos produzem impacto na saúde do indivíduo, assim como em sua qualidade de vida, que segundo a Organização Mundial de Saúde (OMS) é definida como sendo a percepção do indivíduo sobre sua inserção na vida, no contexto cultural e sistemas de valores nos quais ele vive e em relação aos seus objetivos, expectativas, padrões e preocupações, que refletem no bem-estar espiritual, físico, mental, psicológico e emocional, além do convívio social (PEREIRA EF, et al., 2012).

\section{Papel do cirurgião-dentista}

A odontologia está estreitamente ligada à psicologia, desse modo o Cirurgião-Dentista deve possuir uma visão humanista, voltando sua atenção para os aspectos emocionais com 0 intuito de fortalecer emocionalmente o paciente e melhorar o prognóstico do tratamento, para que saiam do consultório mais confiantes, e mantendo um compromisso com os valores éticos e sociais. A obtenção da cooperação do paciente durante o tratamento é de suma importância, e esta pode ser obtida através do estabelecimento de uma relação psicológica com o mesmo (MOTA LQ, et al., 2012).

Os pacientes que possuem seus elementos dentários extraídos experimentam sentimentos que pode levalo a exclusão social e consequente redução da qualidade de vida. Uma pesquisa realizada por Okoje VN, et al. (2018), verificou que após a perda dos dentes, os indivíduos relataram possuir sentimento de tristeza 
(12.9\%), sensação de perda (14\%), depressão (6.4\%) e sensação de envelhecimento (2.3\%). Nepomuceno NVA, et al. (2018), observaram que os impactos na qualidade de vida gerados pela perda dos elementos eram superiores nos indivíduos que não foram reabilitados com algum tipo de prótese.

É importante que o profissional tome conhecimento das oscilações emocionais pelas quais os pacientes passaram da infância até o momento presente, tenha sensibilidade e atenção para a valorização da função e da estética, elementos fundamentais que sustentam a autoestima, e que estabeleça um diálogo com o paciente à fim de construir um vínculo de confiança e um entendimento que irão contribuir para o sucesso do trabalho final (SILVA CR e FERNANDES CAR, 2001).

Diante disso, Guerra CT, et al. (2014) afirma que o exercício da odontologia deve ter uma visão mais humanista e uma responsabilidade muito maior do que somente recuperar apenas a função, a estética e aliviar a dor física do paciente. Ressaltando nesse contexto a importância do papel social do cirurgião-dentista no alerta e no esclarecimento aos pacientes das consequências fisiológicas e dos possíveis impactos na qualidade de vida que a ausência dos elementos dentários pode gerar, sem ignorar o estado psicoemocional dos pacientes.

Assim, o dentista desempenha um importante papel na orientação aos pacientes sobre os efeitos da perda dentária, para que assim este possa melhor se adaptar à nova situação e buscar o tratamento adequado visando o restabelecimento da sua qualidade de vida (GIFT HC e REDFORD M, 2005).

\section{CONSIDERAÇÕES FINAIS}

A ausência dos elementos dentários pode gerar uma série de consequências negativas que influenciam diretamente a qualidade de vida dos indivíduos. As causas mais comuns que levam a essa perda são: doenças bucais, falta de acesso ao sistema, ausência de informação, condição socioeconômica, fatores culturais, e o modelo predominantemente mutilador. Dessa forma, diante dos estudos avaliados, essa revisão de literatura pôde concluir que a perda dos elementos dentários provoca impactos psicológicos e sociais que interferem na qualidade de vida do indivíduo.

\section{REFERÊNCIAS}

1. ASMAR IE. A compensação das consequências do envelhecimento do terço médio e inferior da face em medicina dentária. Dissertação (Mestrado integrado em medicina dentária). Instituto Superior de Ciências da Saúde Ega Moniz. 2017; 83 p.

2. BATISTA MJ, et al. Risk indicators for tooth loss in adult workers. Braz. res oral. São Paulo, 2012; 26(5): 390-396.

3. BORTOLI FR, et al. Percepção da saúde bucal em mulheres com perdas dentárias extensas. Saúde Soc. São Paulo, 2017; 26(2) 533-44.

4. CARNEIRO VFA, et al. Ocorrência de Perda Dentária entre os Usuários da Estratégia de Saúde da Família do Município de Campina Grande - PB. Revista Brasileira de Ciências da Saúde, 2012; 16(2): 137-142.

5. DE SILVA MES, et al. Impacto da perda dentária na qualidade de vida. Ciênc. saúde coletiva, Rio de Janeiro, 2010; 15(3): 841-850.

6. DOMINGOS MÀ. Os impactos biopsicossociais na saúde geral dos adultos gerados pela ausência de saúde bucal MG. Trabalho de Conclusão de Curso (Especialização em Atenção Básica em Saúde da Família). Universidade Federal de Minas Gerais (UFMG), 2012; 32 p.

7. GIFT HC, REDFORD M. Oral Health and the quality of life. Clinic Geriatric Medicine, 2005; 8(53): 673-683.

8. GUERRA CT, et al. Reflexões sobre o conceito de atendimento humanizado em Odontologia. Arch Health Invest. São Paulo, 2014; 6(3): 31-36.

9. IBGE - Instituto Brasileiro de Geografia e estatística. PNS - Pesquisa Nacional de Saúde 2013. Rio de Janeiro, 2015.

10. JORGE TM, et al. Relação entre perdas dentárias e queixas de mastigação, deglutição e fala em indivíduos adultos. Rev. CEFAC, São Paulo, 2009; 11(3): 391-397.

11. MOTA LQ, et al. Humanização no atendimento odontológico: acolhimento da subjetividade dos pacientes atendidos por alunos de graduação em Odontologia. Arq. Odontol. Belo Horizonte, Jul./Set. 2012; 48(3): 151-158.

12. NEPOMUCENDO NVA, et al. Reabilitação protética: sua influência na qualidade de vida. RvACBO, 2019; 28(1): 915. 
13. OKOJE VN, et al. Tooth loss are the patients prepared. Níger J Clin Pract, 2012. 15(2): 172-175.

14. PEREIRA ÉF, et al. Qualidade de vida: abordagens, conceitos e avaliação. Rev. bras. educ. fís. esporte, São Paulo, 2012; 26(2): 241-250.

15. PEREIRA VZ, et al. Avaliação dos Níveis de Ansiedade em Pacientes Submetidos ao Tratamento Odontológico. Revista Brasileira de Ciências da Saúde, 2013; 17(1): 55-64.

16. PROBST LF, et al. Fatores associados aos sentimentos decorrentes da perda dentária total e às expectativas de reposição protética em adultos e idosos. Cad. saúde colet., Rio de Janeiro, 2016; 24(3): 347-354.

17. RIBEIRO CG, et al. Edenbtulism, Severe Tooth Loss and Lack of Functional Dentition in Elders: A Study in Southern Brazil. Braz. Dent. J., Ribeirão Preto, 2016; 27(3): 345-352.

18. SAINTRAIN MV, de SOUZA EH. Impact of tooth loss on the quality of life. Gerodontology, 2012; 29(2).

19. SILVA CR, FERNANDES CAR. Auto-Percepção das avaliações de Saúde Bucal por Idosos. Rev. Saúde Pública, 2001; 35(4): 349-355.

SHULTHEISZ TSV, APRILE MR. Autoestima, conceitos correlatos e avaliação. RECES., 2013; 5(1): 36-48.

20. SOAIGHER KA, et al. O poder da vaidade e do autocuidado na qualidade de vida. Cinergis, Santa Cruz do Sul, 2016; 18(1): 69-72.

21. WOLF RMS. O Significado Psicológico da perda dos dentes em sujeitos adultos. Rev. Assoc. Paul. Cirur. Dent., 1998; v.52, n.4: p.307-316. 